\title{
Stability of Retained Austenite Through a Combined Intercritical Annealing and Quenching and Partitioning (IAQP) Treatment
}

\author{
Ri-Ming Wu $\cdot$ Wei Li $\cdot$ Cheng-Lin Wang $\cdot$ Yi Xiao $\cdot$ Li Wang $\cdot$ Xue-Jun Jin
}

Received: 30 July 2014/Revised: 8 September 2014/Published online: 21 January 2015

(C) The Chinese Society for Metals and Springer-Verlag Berlin Heidelberg 2015

\begin{abstract}
Intercritical annealing (IA) at various temperatures followed by quenching and partitioning (IAQP) treatments was conducted on a cold-rolled $\mathrm{Fe}-0.2 \mathrm{C}-1.42 \mathrm{Si}-1.87 \mathrm{Mn}$ (wt $\%$ ) sheet steel. Optimized microstructure and enhanced mechanical properties were achieved through appropriate adjustment of IA temperatures. The steel which was annealed at $1,033 \mathrm{~K}$ for $600 \mathrm{~s}$, then quenched to $573 \mathrm{~K}$ and partitioned at $693 \mathrm{~K}$ for $20 \mathrm{~min}$, designated as 1033QP steel, exhibits maximum $16.3 \mathrm{vol} \%$ retained austenite (RA) with good mechanical properties (ultimate tensile strength $886 \mathrm{MPa}$ and total elongation 27\%). It was found that the thermal and mechanical stabilities of RA are mainly influenced by the combined effect of its average carbon content and amount of adjacent martensite. Furthermore, the transformation-induced plasticity effect increased the peak $n$-values observed at the second stage of the work hardening of IAQP steels.
\end{abstract}

KEY WORDS: Intercritical annealing and quenching and partitioning treatment (IAQP); Retained austenite (RA); Average carbon content (ACC); Amount of adjacent martensite (AAM); Transformation-induced plasticity (TRIP)

\section{Introduction}

Since transformation-induced plasticity (TRIP) effect was observed by Zackay et al. [1], TRIP steels have attracted renewed interest from both industry and academia [2, 3]. Austenite is retained through a two-step annealing process,

Available online at http://link.springer.com/journal/40195

R.-M. Wu · W. Li · C.-L. Wang · Y. Xiao · X.-J. Jin (ه)

School of Materials Science and Engineering, Shanghai Jiao

Tong University, 800 Dongchuan Rd, Shanghai 200240, China

e-mail: jin@sjtu.edu.cn

R.-M. Wu

School of Materials Science and Engineering, Shanghai

University, 149 Yanchang Rd, Shanghai, China

L. Wang

State Key Lab of Development and Application Technology of Automotive Steels, Baosteel Research Institute,

Shanghai 201900, China i.e., intercritical annealing (IA) followed by bainitic isothermal transformation in low-alloyed steels, and it can enhance ductility of the steels [4]. One of the most innovative procedures to create the microstructures consisting of martensite and retained austenite (RA) is the quenching and partitioning $(\mathrm{Q} \& \mathrm{P})$ process [5], and the amount and stability of the RA in Q\&P steels play important roles on their mechanical properties [6]. In order to produce sufficient amounts of RA in Q\&P steels, a three-step heat treatment was imposed including IA, partitioning Q\&P and final quenching to room temperature [7].

The microstructural evolution during $\mathrm{Q} \& \mathrm{P}$ process is more complex than just the formation of martensite followed by carbon partitioning from martensite to austenite. Examples of this complexity are the formation of epitaxial ferrite during the first quenching step and the formation of bainite, carbides, and carbon gradients as well as migration of martensite/austenite interfaces during the partitioning step [8]. The formation of bainite and carbide is derived from the insufficient thermal stability of RA during 
partitioning [9]. On the other hand, most of the primary austenite transformed into martensite during quenching step, which is harder than austenite retained at room temperature. Therefore, the martensite behaves larger workhardening rate in tension and the incompatibility with the $\mathrm{RA}$, leading to the growing of premature voids at interfaces of martensites and RA, and eventually worsened the plasticity of the steel [10].

Accordingly, ferrite, including equiaxial and bainitic ones, can be used to replace "hard" martensite. For instance, good combinations of tensile strength (1,000-1,200 MPa) and total elongation (14-20\%) were obtained after intercritical annealing and Q\&P (IAQP) treatments by Moor et al. [7]. Gao et al. [11] also improved the mechanical properties of quenching-partitioning-tempering $(\mathrm{Q}-\mathrm{P}-\mathrm{T})$ steel by introducing carbide-free bainite to achieve a product of strength and elongation to $31.4 \mathrm{GPa} \%$. The elongation of Q-P-T steels as reported was raised without a marked sacrifice of strength, and thus the product of strength and elongation was enhanced as a whole. This obvious superiority was achieved by both the improved thermal and mechanical stabilities of RA through excessive carbon partitioning from surrounding equiaxial or bainite ferrite (or carbide-free bainite).

However, precise observations [12] and judicious calculations [13] indicated that there is reluctance of excess carbon within bainite ferrite or martensite in spite of prolonged heat treatment at temperatures where carbon is mobile. Additionally, the inevitable formation of transition carbides during prolonged partitioning step also decreases the amount and stability of RA [14]. These facts demonstrated the relatively weaker effect of bainite ferrite than that of equiaxial ferrite in promoting carbon concentration of RA.

The stability of RA phase in Q\&P steels refers to its resistance to transformation with temperature, stress and strain. One of the factors affecting the stability of RA is the concentration of stabilizing alloying elements in the austenite, and carbon is the most important stabilizing element in low alloy Q\&P steels [15]. Other factors that affect the austenite stability are the size and the morphology of the RA grains, and the property of the surrounding matrix. The mechanical property of the surrounding matrix affects the stress and strain carried by the RA at a given total strain level, as it does in a composite material. For instance, when the austenite phase is surrounded by soft ferrite, it will undergo much global stress and strain than that surrounded by a harder bainite or martensite [10]. Different morphologies of austenite phase also provide the austenite with varying mechanical stabilities [16]. Several morphologies of RA have been observed in Q\&P steels. In the typical Q\&P steel microstructure produced by a three-stage heat treatment, the RA is present as discrete particles between equiaxed ferrite grains. Consequently, the size, shape and composition of the phases surrounding the RA influence its transformation behavior [16].

In order to enhance the TRIP effect and thus mechanical performance of IAQP steels, the present work aims to optimize the $f_{\mathrm{RA}}$ as well as its stability, and further to analyze the correlations between stability of RA and mechanical properties.

\section{Experimental}

The chemical composition of the cold-rolled sheet steel in this investigation is $\mathrm{Fe}-0.20 \mathrm{C}-1.40 \mathrm{Si}-1.87 \mathrm{Mn}$ (wt\%). The microstructures contain ferrite and pearlite as shown in Fig. 1. The $A_{\mathrm{c} 1}, A_{\mathrm{c} 3}$ and $M_{\mathrm{s}}$ temperatures measured by dilatometer are $988,1,136$ and $638 \mathrm{~K}$, respectively.

In order to maximize the volume fraction of austenite at room temperature in IAQP state, the cold-rolled sheet steels were first intercritically annealed at 1,013, 1,033, 1,053 and $1,073 \mathrm{~K}$ for $10 \mathrm{~min}$, followed by Q\&P treatments (i.e.,
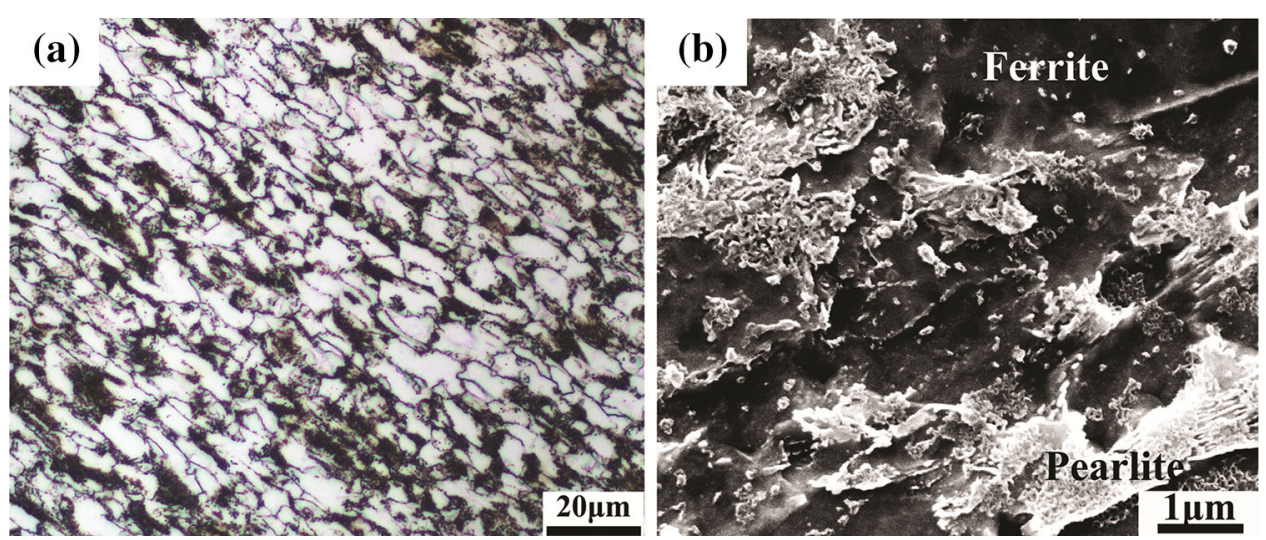

Fig. 1 Optical a SEM b micrographs of as-rolled Fe-0.2C-1.87Mn-1.42Si steel sheet 


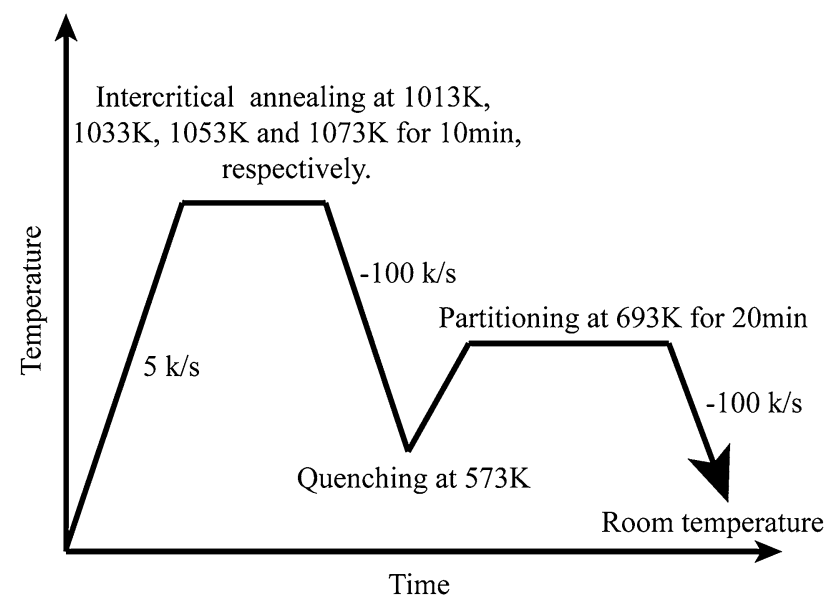

Fig. 2 Schematic diagram of intercritical annealing and quenching and partitioning (IAQP) treatments

quenched to $T_{\mathrm{Q}}=573 \mathrm{~K}$ and partitioned at $T_{\mathrm{P}}=693 \mathrm{~K}$ for $20 \mathrm{~min}$ ), and then quenched into water at room temperature. These treated samples were designated as 1013QP, 1033QP, 1053QP and 1073QP steels, respectively. The heat treatment processes were illustrated in Fig. 2.

Field emission gun scanning electron microscope (FEGSEM) observations were performed on a JEOL JSM-7600F operating at $15 \mathrm{kV}$. The $f_{\mathrm{RA}}$ and its $\mathrm{ACC}$ of RA were determined by $\mathrm{X}$-ray diffraction (XRD) with $\mathrm{Cu}-\mathrm{K} \alpha$ radiation on a D/max-2550 X-ray diffractometer at room temperature, based on a direct comparison of the integrated intensity of $(200)_{\gamma},(220)_{\gamma}$ and (311) $)_{\gamma}$ with $(200)_{\alpha}$ and $(211)_{\alpha}$ peaks [17]. The lattice parameter of the austenite phase was determined from a fit of the peak positions of the monitored powder reflections to Bragg's law. The changes in lattice parameter of the austenite phase reflect the difference in the average interstitial carbon concentration for IAQP steels. At room temperature, the compositional dependence of the austenite lattice parameter $a_{\gamma}$ is given by Eq. (1) $[18,19]$.

$a_{\gamma}=3.556+0.0453 x_{\mathrm{C}}+0.00095 x_{\mathrm{Mn}}+0.0056 x_{\mathrm{Al}}$

where $a_{\gamma}$ is in $\stackrel{\AA}{;} x_{\mathrm{C}}, x_{\mathrm{Mn}}, x_{\mathrm{Al}}$ are the contents of $\mathrm{C}, \mathrm{Mn}$ and $\mathrm{Al}$ which in wt\%. The influence of $\mathrm{Si}, \mathrm{Mn}$ and $\mathrm{Al}$ was found to be negligible within the experimental accuracy [19]. The average carbon content of austenite was estimated by Eq. (1).

Electron backscatter diffraction (EBSD) measurement was carried out on the IAQP sample surface parallel to the rolling direction. The constituent phase was discriminated by processing of the EBSD data with Channel 5 software provided by HKL Technology (Oxford Instruments).

Differential scanning calorimetry (DSC) was used to analyze the phase transformation of RA in steels upon heating and cooling [20]. The samples were cut into disks $(\phi$
$3.5 \mathrm{~mm} \times 1.3 \mathrm{~mm})$. One set of samples were heated from 323 to $823 \mathrm{~K}$ at a heating rate of $20 \mathrm{~K} / \mathrm{min}$ in DSC chamber and then cooled to room temperature at a rate of $20 \mathrm{~K} / \mathrm{min}$. Another set of samples were cooled down to about $123 \mathrm{~K}$ and then heated to room temperature at a rate of $20 \mathrm{~K} / \mathrm{min}$. Baselines were obtained by repeating the heating or cooling procedures with the samples in DSC chamber.

Tensile samples in width of $5 \mathrm{~mm}$ and thickness of $1.2 \mathrm{~mm}$ with a gauge length of $15 \mathrm{~mm}$ were machined from the sheet steel. The rolling direction was selected as the tensile direction.

The plastic flow behavior of most metals and alloys can be described by the Hollomon relation [21] as follows: $\sigma=k \varepsilon^{n}$

where $k$ and $n$ are fitting constants usually termed as strainhardening coefficient and strain-hardening exponent, respectively. The value of $n$ correlates the slope of the true stress versus true strain curve $(n=(\varepsilon / \sigma) \times(\mathrm{d} \sigma / \mathrm{d} \varepsilon))$, where $(\mathrm{d} \sigma / \mathrm{d} \varepsilon)$ is the rate of work hardening, which reflects the ability to resist localization during deformation.

Mechanical stability of RA was studied by intermittently measuring the $f_{\mathrm{RA}}$ by XRD method after attainment of various engineering strains $(0,1 \%, 5 \%, 10 \%, 15 \%$ and fracture) during tensile tests.

\section{Results and Discussion}

\subsection{Microstructure}

Figure 3 shows the SEM micrographs of 1013QP, 1033QP, 1053QP and 1073QP steels. They mainly consist of ferrite and islands of martensite/austenite (M/A). Besides, 1013QP steel contains a small fraction of carbides, while 1053QP and 1073QP steels contain bainite (B) and tempered martensite (TM). Figure 4 is the EBSD maps of the IAQP steels, in which red, yellow and green colors indicate RA, carbide and ferrite/martensite, respectively. The measured volume fractions of the constituent phases in different IAQP-treated steels determined by quantitative metallography combined with XRD are shown in Fig. 5.

It can be seen that the amount of M/A increases as the $T_{\mathrm{A}}$ increases from 1,013 to $1,073 \mathrm{~K}$. About $1.6 \mathrm{vol} \%$ of carbide in 1013QP steel indicates that annealing at $1013 \mathrm{~K}$ for $10 \mathrm{~min}$ is not enough for the dissolution.

As shown in Fig. 5, the fraction of bainite $\left(f_{\mathrm{B}}\right)$ in $1053 \mathrm{QP}$ was $2 \mathrm{vol} \%$ and increased to $12.8 \mathrm{vol} \%$ for $1073 \mathrm{QP}$ steel. Meanwhile, the amount of tempered martensite $\left(f_{\mathrm{TM}}\right)$ was also increased from 6 to $20 \mathrm{vol} \%$ (Fig. 5a, b). Both the $f_{\mathrm{B}}(2 \mathrm{vol} \%$ ) and $f_{\mathrm{RA}}(7.2 \mathrm{vol} \%)$ in 1053QP steel are much lower than those (12.8 and $9.8 \mathrm{vol} \%$ ) in 1073QP steel. 

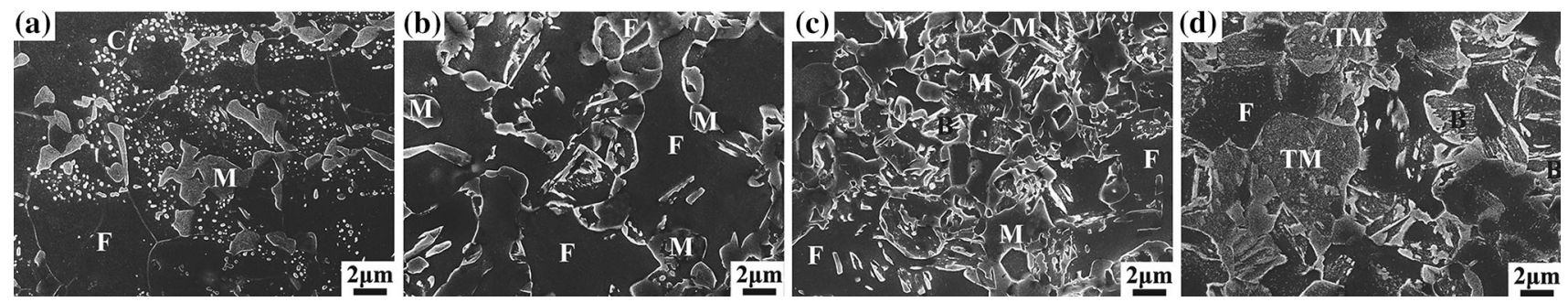

Fig. 3 SEM micrographs of IAQP steels, C, F, B, M and TM indicate carbide, ferrite, bainite, martensite and tempered martensite, respectively: a 1013QP steel; b 1033QP steel; c 1053QP steel; d 1073QP steel
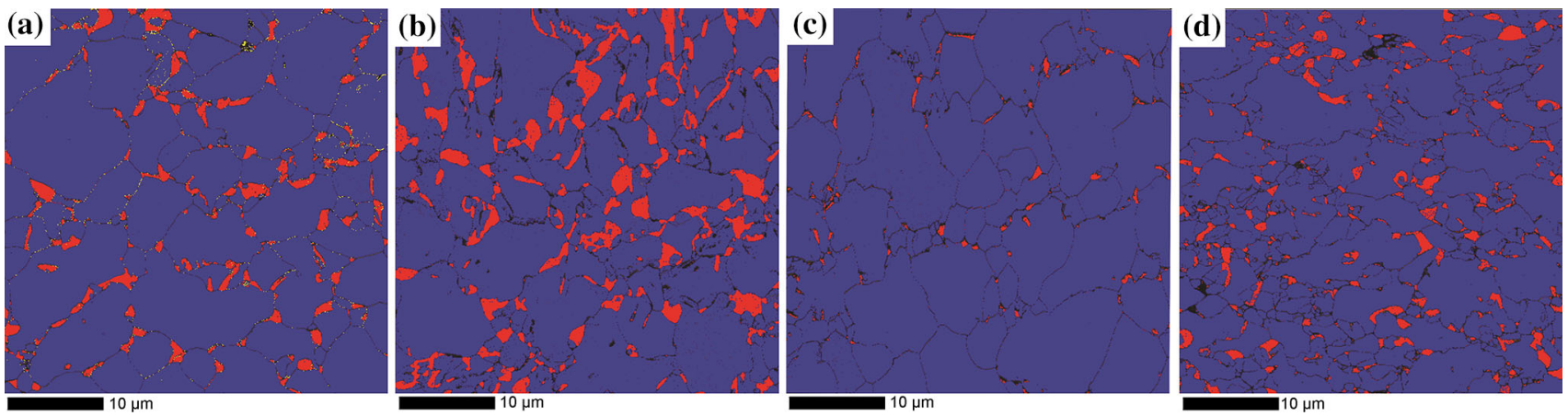

Fig. 4 EBSD maps of IAQP steels, blue, yellow and red colors indicate ferrite, cementite, and austenite, respectively. Dark areas correspond to a very low band contrast: a 1013QP steel; b 1033QP steel; c 1053QP steel; d 1073QP steel

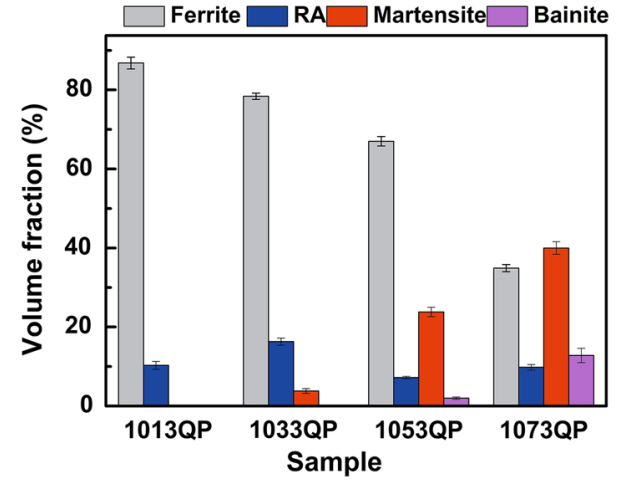

Fig. 5 Measured volume fractions of phase constituents in IAQP steels by quantitative metallography combined with XRD

Consequently, not only the undissolved carbide in 1013QP steel, but also the martensite and bainite in 1053QP and 1073QP steels are responsible for reducing the carbon resource which could be enriched into RA in those samples. It might be also reasonable to conversely interpret the reason why $1033 \mathrm{QP}$ steel contains the largest $f_{\mathrm{RA}}$.

\subsection{Mechanical Properties}

Figure 6a shows the curves of engineering stress versus engineering strain for IAQP samples, while the true stress versus true strain curves of IAQP samples are presented in
Fig. 6b. It can be seen from Fig. 6a that ultimate tensile strengths remain approximately the same when the annealing temperature is increased from 1,013 to $1,073 \mathrm{~K}$.

TRIP effect plays another positive role in keeping high ultimate tensile strengths of the 1013QP and 1033QP steels containing only few amounts of martensite and, more importantly, it enhances the total elongation of 1013QP steel to the largest $29.5 \%$. The yield strengths of 1013QP or 1033 QP steel are lower than that of 1053QP steel due to less amount of harder martensite, and the yield strength and ultimate tensile strength of 1073QP steel are highest and they have very low elongations because of their largest amounts of martensite, as shown in Fig. 5. That is to say, for these four IAQP steels, 1033QP steel exhibits best combination of mechanical property with ultimate tensile strength of $886 \mathrm{MPa}$ and total elongation of $29.5 \%$.

It is worth mentioning that the RA at the third stage of work hardening shown in Fig. $6 \mathrm{c}$ is more stable, because after a certain amount of deformation, the tangled dislocations in RA would suppress martensitic transformation [22], which known as mechanical stabilization [23], and it help to resist the onset of TRIP effect till the later stage of deformation.

\subsection{Thermal Stability of RA}

The thermal stability of RA in IAQP steels can be directly revealed by DSC on the basis of exothermic reactions due 

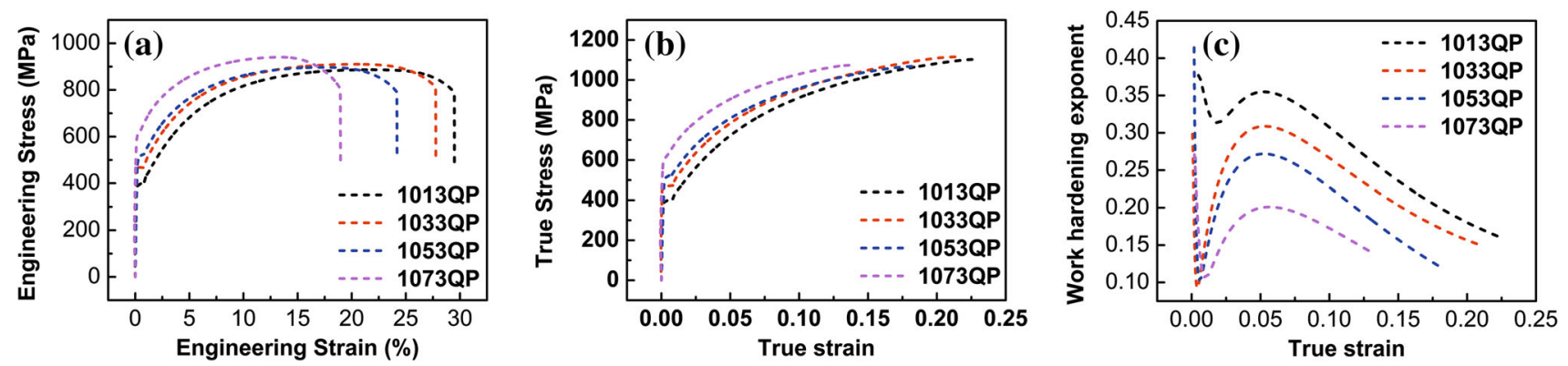

Fig. 6 Tensile mechanical properties of IAQP steels: a engineering stress versus engineering strain curves; $\mathbf{b}$ true stress versus true strain curves; c work-hardening exponent versus true strain
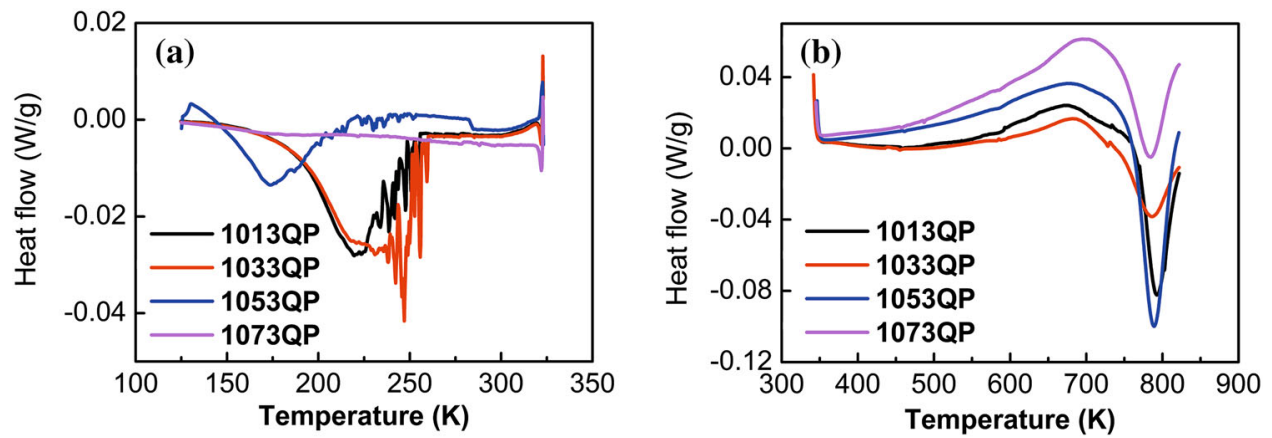

Fig. 7 Exothermic peaks (absolute value) of martensitic transformation a decomposition $\mathbf{b}$ of retained austenite upon cooling and heating processes, respectively

Table 1 Peak temperature of martensitic transformation $\left(T_{\mathrm{Ms}}\right)$ and decomposition temperature of retained austenite $\left(T_{\mathrm{RA}}\right)$ and the average carbon content (ACC) of retained austenite of the IAQP steels

\begin{tabular}{lcll}
\hline Steel & $T_{\mathrm{Ms}}(\mathrm{K})$ & $T_{\mathrm{RA}}(\mathrm{K})$ & Carbon content (at\%) \\
\hline 1013QP & 223 & 795 & 1.23 \\
$1033 \mathrm{QP}$ & 223 & 790 & 1.42 \\
$1053 \mathrm{QP}$ & 183 & 791 & 1.33 \\
$1073 \mathrm{QP}$ & $<\mathbf{1 2 3}$ & 785 & 1.61 \\
\hline
\end{tabular}

to the transformations of RA. Figure 7a shows the exothermic peaks of martensitic transformation for the IAQP samples displayed by DSC upon cooling and they are all below ambient temperature. Then, the peak temperature of martensitic transformation can be approximately determined as listed in Table 1. The peak temperatures of martensitic transformation of RA in 1013QP, 1033QP and 1053QP steels are 223, 223 and $183 \mathrm{~K}$, respectively. However, the peak temperature of martensitic transformation of RA in 1073QP steel is probably out of the measurable range. Figure $7 \mathrm{~b}$ shows the exothermic peaks of RA decomposition by DSC upon heating. It can be seen that the decomposition of RA occurs in a relatively narrow temperature range, which indicates there is no evident correlation between the temperature of austenite decomposition and the thermal stability of austenite above ambient temperature.

The stability of RA is reflected by the start temperature of martensitic transformation $\left(M_{\mathrm{s}}\right)$; ACC is the most predominant factor influencing $M_{\mathrm{s}}$. Of course, there are other factors affecting the stability of retained austenite such as size, morphology and surrounding phase [24], but the differences in size and morphology are small in the as-tailored IAQP steels. Therefore, the surrounding phase is another factor to be particularly concerned. Because the RA in IAQP steels is mostly surrounded by martensite, i.e., the morphology of $\mathrm{M} / \mathrm{A}$, the amount of adjacent martensite (AAM) is considered. Here the AAM is determined as the total amount of TM (tempered martensite) and UTM (untempered martensite). It can be seen from Fig. 5 that 1033QP steel has 3.8 vol\% AAM, which is far lower than that of 1053QP steel (23.8 vol\%). Figure 8 shows the typical TEM images of 1033QP steel where the blocky RA was surrounded by adjacent martensite.

Figure 9 is the XRD patterns of IAQP samples slowly scanned in a $2 \theta$ range of $74^{\circ}-76^{\circ}$, which shows their $(220)_{\gamma}$ peaks of austenite. A Gaussian multipeak fitting method was employed. The different peak positions of $(220)_{\gamma}$ can be attributed to the ACC of RA, which can be qualitatively determined as shown in Table 1. A lower ACC in RA is 

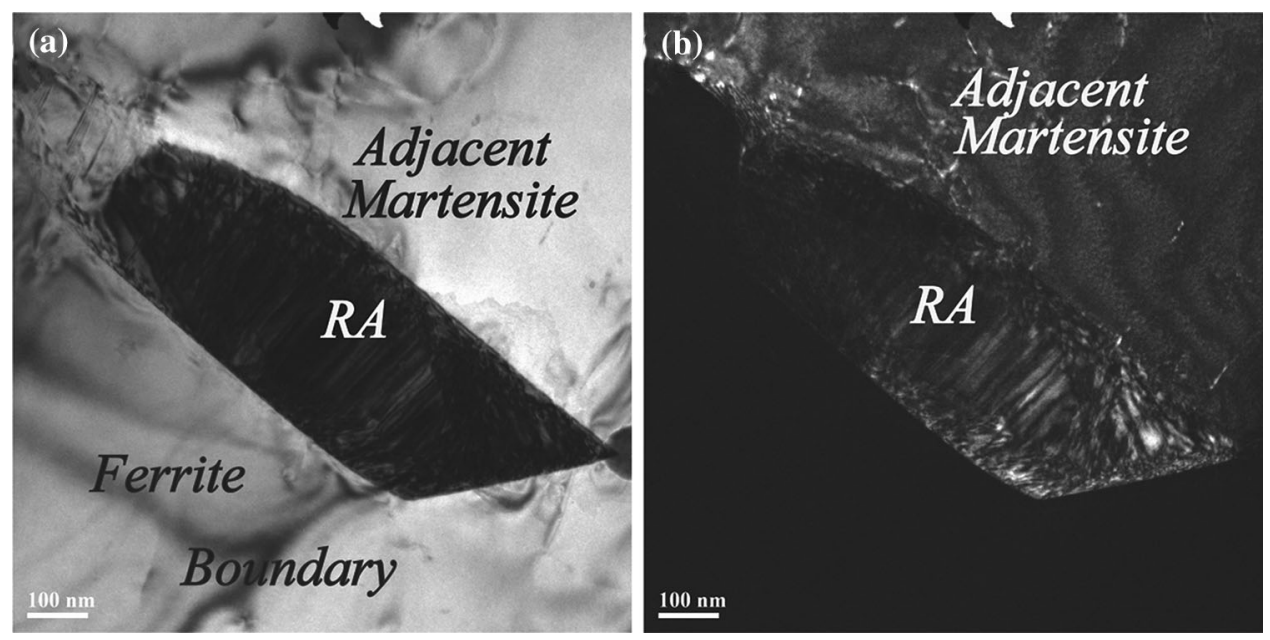

Fig. 8 TEM images of 1033QP steel: a bright field image; b dark field image

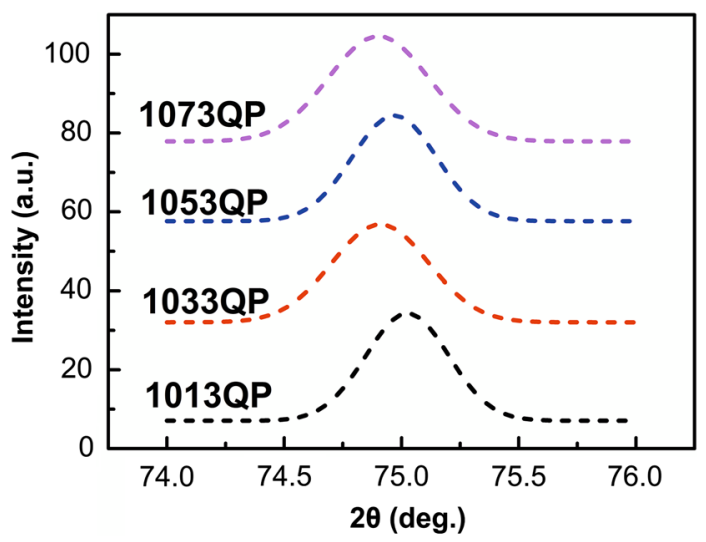

Fig. 9 (220) peaks of austenite in IAQP steels ( $2 \theta$ ranges from $74^{\circ}$ to $76^{\circ}$ ) from XRD patterns

related to a higher $2 \theta$ of an austenite (220) peak. Considering that a lower peak temperature of martensitic transformation indicates a higher stability of RA, the peak temperature of martensitic transformation listed in Table 1 implies that the RA in 1053QP is more stable than that in $1033 \mathrm{QP}$, although the RA has a lower ACC.

It can be deduced that the higher AAM of RA in 1053QP steel exhibits a better stability of RA than that of 1033QP steel. This clearly confirms that the AAM of RA in IAQP steels plays a dominant role in stabilizing RA. Some studies $[24,25]$ also reveal that the existing hard martensite adjacent to RA helps to reduce the amount of stress and strain should be carried by RA, and it would beneficially increase the stability of RA. Another similar mechanism (martensite shielding effect) was proposed by Jacques et al. [26], i.e., martensite with high yield stress can "shield" the austenite during deformation. This explains that the AAM of RA in IAQP steels plays a dominant role in stabilizing RA.

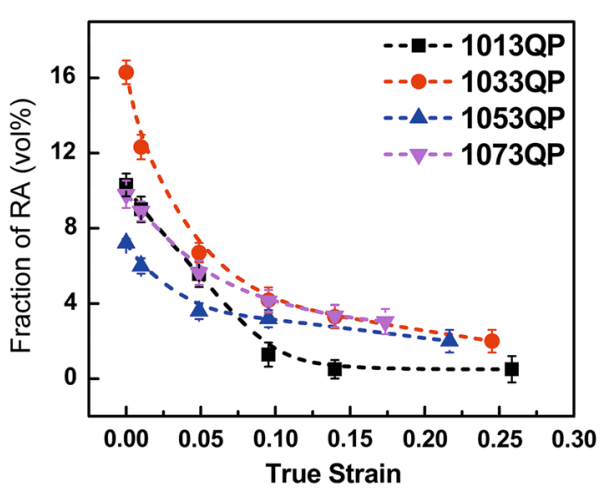

Fig. 10 Volume fraction of retained austenite $\left(f_{\mathrm{RA}}\right)$ in IAQP steels as a function of applied true strain

\subsection{Mechanical Stability of RA}

The mechanical stability of RA and strain-induced transformation to martensite governs the strain-hardening behavior and ductility. The strain-hardening rate is increased by transformation of austenite into martensite and necking is delayed [27].

Figure 10 shows the $f_{\mathrm{RA}}$ in IAQP steels as a function of true strain upon tensile tests. It is found that about approximately two-thirds of RA transformed into martensite below the true strain about 0.10 for all these IAQP samples. The mechanical stability of RA reflected by the martensitic transformation rate reduces as the annealing temperature decreases, and the RA in 1013QP steel exhibits the lowest mechanical stability.

The deformation-induced martensitic transformation of RA in IAQP steels can be directly correlated to the TRIP effect of RA as well as the mechanical stability (i.e., transformation rate) of RA. The XRD measurements revealed that the austenite fraction decreases monotonically 
Table 2 Decreasing rate of austenite during tension $\left(\mathrm{d} f_{\mathrm{M}} / \mathrm{d} \varepsilon\right)$, flow stress of IAQP steels $\left(\sigma_{\mathrm{f}}\right)$, work-hardening rate of martensite at strain of $0.05\left(\mathrm{~d} \sigma_{\mathrm{M}} / \mathrm{d} \varepsilon_{\varepsilon=0.05}\right)$, and the flow stresses of martensite and austenite $\left(\sigma_{\mathrm{M}}\right.$ and $\left.\sigma_{\mathrm{RA}}\right)$ for different steels [30]

\begin{tabular}{llllll}
\hline Steel & $\mathrm{d} f_{\mathrm{M}} / \mathrm{d} \varepsilon$ & $\begin{array}{l}\sigma_{\mathrm{f}} \\
(\mathrm{MPa})\end{array}$ & $\begin{array}{l}\mathrm{d} \sigma_{\mathrm{M}} / \mathrm{d} \varepsilon_{\varepsilon=0.05} \\
(\mathrm{MPa})\end{array}$ & $\begin{array}{l}\sigma_{\mathrm{M}} \\
(\mathrm{MPa})\end{array}$ & $\begin{array}{l}\sigma_{\mathrm{RA}} \\
(\mathrm{MPa})\end{array}$ \\
\hline $1013 \mathrm{QP}$ & 1.10 & 386 & 1,500 & 2,400 & 600 \\
$1033 \mathrm{QP}$ & 1.15 & 469 & 1,500 & 2,400 & 600 \\
$1053 \mathrm{QP}$ & 1.00 & 514 & 1,500 & 2,400 & 600 \\
$1073 \mathrm{QP}$ & 0.80 & 606 & 1,500 & 2,400 & 600 \\
\hline
\end{tabular}

with true tensile strain as plotted in Fig. 10, which can be simulated well by the exponent decay law as follows [28]:

$f_{\gamma}=f_{\gamma 0} \exp (-k \varepsilon)$

where $f_{\gamma}, f_{\gamma 0}$ and $k$ are the austenite fraction at true strain $\varepsilon$, the initial austenite fraction and the mechanical stability of austenite, respectively.

The work-hardening curves can be divided into three stages [27]. In stage I, $n$ decreases as the applied stress, thus the true strain, increases before yielding. In stage II, after a shortly decrement, $\mathrm{n}$ begins increasing to a peak value, and this rebound is contributed to the TRIP effects, which will be testified afterward. In stage III, $n$ decreases progressively with increasing strain until failure. The RA in this stage may be more stable, because most of RA with lower stability is transformed in the previous two stages and only stable austenite remains. In order to understand the three-stage work-hardening behavior as observed in Fig. $6 \mathrm{c}$, the flow stress $\sigma_{\mathrm{f}}$ of multiphase steel could be formulated by a mixed hardening law proposed by Embury and Bouaziz [29]:

$\sigma_{f}=f_{\alpha} \sigma_{\alpha}+f_{\gamma} \sigma_{\gamma}+\left(1-f_{\alpha}-f_{\gamma}\right) \sigma_{M}$

$\mathrm{d} f_{M} / \mathrm{d} \varepsilon=-\mathrm{d} f_{\gamma} / \mathrm{d} \varepsilon$

where $\sigma_{\alpha}, \sigma_{\gamma}$ and $\sigma_{\mathrm{M}}$ are the flow stresses, $f_{\alpha}, f_{\gamma}$ and $f_{\mathrm{M}}$ are the volume fractions of ferrite, austenite and deformationinduced martensite, respectively.

The "instantaneous" work-hardening rate varying with the true strain $\varepsilon$ could be derived as:

$$
\begin{aligned}
\mathrm{d} \sigma_{f} / \mathrm{d} \varepsilon= & f_{\alpha} \times \mathrm{d} \sigma_{\alpha} / \mathrm{d} \varepsilon+f_{\gamma} \times \mathrm{d} \sigma_{\gamma} / \mathrm{d} \varepsilon+f_{M} \times \mathrm{d} \sigma_{M} / \mathrm{d} \varepsilon \\
& +\mathrm{d} f_{M} / \mathrm{d} \varepsilon \times\left(\sigma_{M}-\sigma_{\gamma}\right) .
\end{aligned}
$$

The instantaneous work-hardening exponent $n_{\text {inst }}$ can be described as follows:

$n_{\text {inst }}=\left(\varepsilon / \sigma_{f}\right) \times \mathrm{d} \sigma_{f} / \mathrm{d} \varepsilon$

At stage I $(0<\varepsilon<0.01)$, the decreasing rates of retained austenite of IAQP steels were small (Fig. 10), because the retained austenite in as-tailored IAQP steels is relatively stable at lower stress or stain. The contribution of

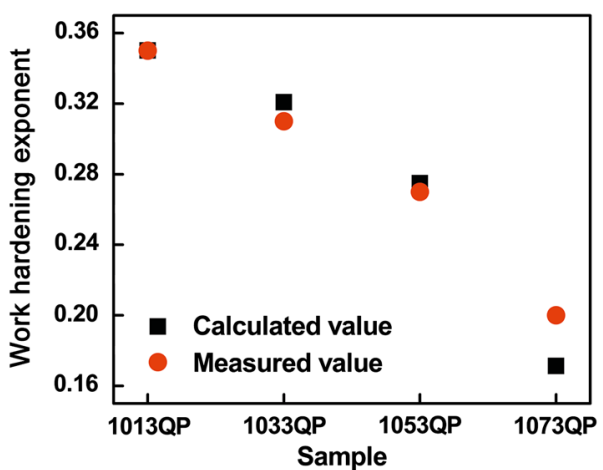

Fig. 11 Calculated and measured instantaneous work-hardening exponents of IAQP steels when $\varepsilon=0.05$

work hardening from deformation-induced martensite was considered to be little, so

$\mathrm{d} f_{\gamma} / \mathrm{d} \varepsilon \approx 0$

$n_{\text {inst }} \approx\left(\varepsilon / \sigma_{f}\right) \times\left[f_{\alpha} \times \mathrm{d} \sigma_{\alpha} / \mathrm{d} \varepsilon+f_{\gamma} \times \mathrm{d} \sigma_{\gamma} / \mathrm{d} \varepsilon\right]$.

With increasing $\varepsilon$, the contributions to $n_{\text {inst }}$ from ferrite and austenite decrease sharply. In stage II $(0.01<\varepsilon<0.05)$, $\sigma_{\alpha}$ is relatively small, and thus the work-hardening rate is contributed mainly from the dislocation hardening of transformed martensite and austenite,

$n_{\text {inst }} \approx\left(\varepsilon / \sigma_{f}\right) \times\left[f_{M} \times \mathrm{d} \sigma_{M} / \mathrm{d} \varepsilon+\mathrm{d} f_{M} / \mathrm{d} \varepsilon \times\left(\sigma_{M}-\sigma_{\gamma}\right)\right]$.

The instantaneous $\mathrm{d} f_{M} / \mathrm{d} \varepsilon$ at $\varepsilon=0.05$ for transformed martensite can be obtained by differentiating the curves, as shown in Fig. 10. The increasing work-hardening rate of transformed martensite at strain 0.05 , flow stress of IAQP steels, work-hardening rate of martensite, and the flow stress of martensite and austenite the values of are shown in Table 2 [30]. The measured values of the $n$ values shown in Fig. 11 are quite closely to that happened at the true strain of 0.05 as it was found in Fig. 6c. That is to say, the deformation-induced martensite transformation gives rise to the peak $n$ values at $\varepsilon=0.05$ and they are very near to the peaks as well. Therefore, the contributions of workhardening exponent from transformed martensite play a crucial role in strengthening.

In stage III $\left(0.05<\varepsilon<\varepsilon_{\mathrm{t}}\right), \varepsilon_{\mathrm{t}}$ is the total strain at fracture of the tested steels, $\sigma_{\gamma}$ and $\sigma_{\mathrm{M}}$ could remain the same, but $\mathrm{d} f_{\mathrm{M}} / \mathrm{d} \varepsilon$ decreases dramatically to lower than 0.2 , which implies that the TRIP effect becomes weak and the workhardening exponent decreases gradually as shown in Fig. 6c

Recently, Challa et al. [27] also confirm that TRIP effect gives rise to a peak value in the second stage of workhardening exponent curves in nano- or ultra-fine-grained stainless steels, and the excellent properties were achieved 
by refining the grain size and thus changing the active energy of strain-induced martensitic transformation. Similarly, a high volume fraction of acicular RA was obtained in low-alloyed steels by Ding et al. [31], and the superior mechanical properties were ascribed to the adjustment of morphology of RA. Therefore, the stability of the RA in IAQP steels and the TRIP effects play significant roles in the work-hardening behavior, and consequently their mechanical properties.

\section{Conclusions}

Intercritical annealing followed by quenching \& partitioning treatments (IAQP) were conducted on a cold-rolled $\mathrm{Fe}-0.2 \mathrm{C}-1.42 \mathrm{Si}-1.87 \mathrm{Mn}$ (wt $\%$ ) sheet steel. Steel annealed at $1,033 \mathrm{~K}$ for $10 \mathrm{~min}$, and then quenched to $573 \mathrm{~K}$ and partitioned at $693 \mathrm{~K}$ for $20 \mathrm{~min}$, shows a maximum retained austenite $(16.3 \mathrm{vol} \%)$ and a good combination of mechanical properties (ultimate tensile strength of $886 \mathrm{MPa}$ and total elongation of 27\%).

It was found that the thermal and mechanical stabilities of RA are increased by increasing the amount of the average carbon content and the amount of adjacent martensite.

The TRIP effect gives rise to a peak value in the second stage $(0.01<\varepsilon<0.05)$ of the work hardening of IAQP steels. By controlling RA with appropriate stability in IAQP steels can optimize the TRIP effect and achieve desirable mechanical properties.

Acknowledgments This work was financially supported by the National Natural Science Foundation of China (No. 51174251) and the National Basic Research Program of China (No. 2010CB630803). The authors would like to acknowledge Professor David Embury, University of McMaster, Ontario, Canada, for helpful discussion. The authors acknowledge Professor Shipu Chen, Shanghai Jiao Tong University, Shanghai, China, for helpful discussion.

\section{References}

[1] P. Guo, J. Jiang, S. Shen, L. Guo, Int. J. Hydrogen Energy 38, 13097 (2013)

[2] O. Matsumura, Y. Sakuma, H. Takechi, Trans. Iron Steel Inst. Jpn. 27, 570 (1987)
[3] O. Matsumura, Y. Sakuma, H. Takechi, Scr. Metall. 21, 1301 (1987)

[4] R. Zhu, S. Li, I. Karaman, R. Arroyave, T. Niendorf, H.J. Maier, Acta Mater. 60, 3022 (2012)

[5] J.G. Speer, A.M. Streicher, D.K. Matlock, F. Rizzo, G. Krauss, in Quenching and Partitioning: A Fundamentally New Process to Create High Strength Trip Sheet Microstructures, ed. by M. Merwin, E. B. Damm. Austenite Formation and Decomposition (Wiley, NJ, 2003)

[6] H. Li, X. Lu, W. Li, X. Jin, Metall. Mater. Trans. A 41, 1284 (2010)

[7] E.D. Moor, J.G. Speer, D.K. Matlock, J.H. Kwak, S.B. Lee, J. Iron Steel Inst. 51, 137 (2011)

[8] M. Santofimia, L. Zhao, J. Sietsma, Metall. Mater. Trans. A 42, $3620(2011)$

[9] M. Santofimia, L. Zhao, J. Sietsma, Metall. Mater. Trans. A 40, 46 (2009)

[10] P.J. Jacques, Acta Mater. 56, 3900 (2008)

[11] G. Gao, H. Zhang, Z. Tan, W. Liu, B. Bai, Mater. Sci. Eng., A 559, 165 (2013)

[12] F.G. Caballero, M.K. Miller, S.S. Babu, C. Garcia-Mateo, Acta Mater. 55, 381 (2007)

[13] A. Mesbah, F. Zaïri, M. Naït-Abdelaziz, J.M. Gloaguen, K. Anoukou, A. Zaoui, Z. Qu, T. Boukharouba, J.M. Lefebvre, Compos. Sci. Technol. 101, 71 (2014)

[14] M. Santofimia, L. Zhao, R. Petrov, J. Sietsma, Mater. Charact. 59, 1758 (2008)

[15] P. Jacques, Curr. Opin. Solid State Mater. Sci. 8, 259 (2004)

[16] I. Timokhina, P. Hodgson, E. Pereloma, Metall. Mater. Trans. A 35, 2331 (2004)

[17] R. Miller, Trans. ASM 57, 892 (1964)

[18] L. Cheng, N. Van Der Pers, A. Böttger, T.H. De Keijser, E. Mittemeijer, Metall. Trans. A 21, 2857 (1990)

[19] D. Dyson, B. Holmes, J. Iron Steel Inst. 208, 469 (1970)

[20] P. Morra, A. Böttger, E. Mittemeijer, J. Therm. Anal. Calorm. 64, 905 (2001)

[21] J.H. Hollomon, AIME Trans. 12, 1 (1945)

[22] L. Zhang, S. Takahashi, Y. Kamada, Scr. Mater. 57, 711 (2007)

[23] J.F. Breedis, Acta Metall. 13, 239 (1965)

[24] X. Xiong, B. Chen, M. Huang, J. Wang, L. Wang, Scr. Mater. 68, 321 (2012)

[25] H. Luo, Scr. Mater. 66, 829 (2012)

[26] P. Jacques, F. Delannay, J. Ladrière, Metall. Mater. Trans. A 32, 2759 (2001)

[27] V.S.A. Challa, X.L. Wan, M.C. Somani, L.P. Karjalainen, R.D.K. Misra, Scr. Mater. 86, 60 (2014)

[28] K.I. Sugimoto, M. Kobayashi, S.I. Hashimoto, Metall. Mater. Trans. A 23, 3085 (1992)

[29] D. Embury, O. Bouaziz, Annu. Rev. Mater. Res. 40, 213 (2010)

[30] J. Shi, X. Sun, M. Wang, W. Hui, H. Dong, W. Cao, Scr. Mater. 63, 815 (2010)

[31] R. Ding, D. Tang, A. Zhao, Scr. Mater. 88, 21 (2014) 